\title{
Variational Analysis of a Frictional Contact Problem with Wear and Damage
}

\author{
Mohammed Salah Mesai Aoun ${ }^{a}$, Mohamed Selmani ${ }^{b}$ and \\ Abdelaziz Azeb Ahmed ${ }^{c}$ \\ a \\ ${ }^{a}$ Department of Mathematics, University of Biskra \\ 07000 Biskra, Algeria \\ ${ }^{b}$ Laboratory of Applied Mathematics, Faculty of Sciences, University Ferhat \\ Abbas of Stif1 \\ 19000 Setif, Algeria \\ ${ }^{c}$ Laboratory of Operator Theory and PDE: Foundations and Applications, \\ Faculty of Exact Sciences, University of El Oued \\ 39000 El Oued, Algeria \\ E-mail: mesaisalah1977@gmail.com \\ E-mail: mohamed.selmani@univ-setif.dz \\ E-mail(corresp.): aziz-azebahmed@univ-eloued.dz
}

Received January 20, 2020; revised December 20, 2020; accepted December 20, 2020

\begin{abstract}
We study a quasistatic problem describing the contact with friction and wear between a piezoelectric body and a moving foundation. The material is modeled by an electro-viscoelastic constitutive law with long memory and damage. The wear of the contact surface due to friction is taken into account and is described by the differential Archard condition. The contact is modeled with the normal compliance condition and the associated law of dry friction. We present a variational formulation of the problem and establish, under a smallness assumption on the data, the existence and uniqueness of the weak solution. The proof is based on arguments of parabolic evolutionary inequations, elliptic variational inequalities and Banach fixed point.
\end{abstract}

Keywords: quasistatic process, electro-viscoelastic materials, damage, normal compliance, friction, wear, existence and uniqueness, fixed point arguments, weak solution.

AMS Subject Classification: 35J85; 49J40; 47J20; 74M1.

Copyright (c) 2021 The Author(s). Published by Vilnius Gediminas Technical University This is an Open Access article distributed under the terms of the Creative Commons Attribution License (http://creativecommons.org/licenses/by/4.0/), which permits unrestricted use, distribution, and reproduction in any medium, provided the original author and source are credited. 


\section{Introduction}

The piezoelectric effect is characterized by the coupling between the mechanical and electrical behavior of the materials. It consists on the apparition of electric charges on the surfaces of some crystals after their deformation. Conversely, it was proved experimentally that the action of electric field on the crystals may generate strain and stress. A deformable material which presents such a behavior is called a piezoelectric material. Piezoelectric materials are used extensively as switches and actuators in many engineering systems, in radioelectronics, electroacoustics and measuring equipments. However, there are very few mathematical results concerning contact problems involving piezoelectric materials and therefore there is a need to extend the results on models for contact with deformable bodies which include coupling between mechanical and electrical properties. General models for elastic materials with piezoelectric effects can be found in $[2,7]$. A static frictional contact problem for electric-elastic materials was considered in $[3,10,12]$. A slip-dependent frictional contact problem for electro-elastic materials was studied in [21]. Contact problems with friction or adhesion for electro-viscoelastic materials were studied in $[4,9,15,20,22,27]$ and recently in [8] for the case of an electrically conductive foundation. For works concerned with the frictional contact problems for electro-viscoelastic materials with long memory, we refer to $[15,16]$ and the references therein. In the present paper we consider a mathematical model for the process of a frictional contact problem with normal compliance and wear for an electro-viscoelastic material with long memory, damage and a moving conductive foundation. The foundation is assumed to move steadily and only sliding contact takes places. A mathematical models which describe the equilibrium of an elastic or a viscoelastic body in frictional contact with a moving foundation were considered in $[24,25,26]$. In all these papers, the damage function $\beta$ is restricted to have values between zero and one. When $\beta=1$ there is no damage in the material, when $\beta=0$ the material is completely damaged, when $0<\beta<1$ there is partial damage and the system has a reduced load carrying capacity. Contact problems with damage have been investigated in $[14,17,18,23]$.

The rest of the paper is structured as follows. In Section 2, we present the notation and some preliminaries. In Section 3, we present the mechanical problem, we list the assumptions on the data and give the variational formulation of the problem. In Section 4, we state our main existence and uniqueness result. It is based on arguments of classical results for elliptic variational inequalities, on parabolic inequalities and fixed point arguments.

\section{Notation and preliminaries}

In this section we present the notation we shall use and some preliminary material. For further details, we refer the reader to [5]. We denote by $\mathbb{S}^{d}$ the space of second order symmetric tensors on $\mathbb{R}^{d}(d=2,3)$, while "." and | . | will represent the inner product and the Euclidean norm on $\mathbb{S}^{d}$ and $\mathbb{R}^{d}$. Let $\Omega \subset \mathbb{R}^{d}$ be a bounded domain with a Lipschitz boundary $\Gamma$ and let $\boldsymbol{\nu}$ denote the unit outer normal on $\Gamma$. Everywhere in the sequel the index $i$ and $j$ run from 1 to $d$, 
summation over repeated indices is implied and the index that follows a comma represents the partial derivative with respect to the corresponding component of the independent spatial variable. We use the standard notation for Lebesgue and Sobolev spaces associated to $\Omega$ and $\Gamma$ and introduce the spaces

$$
\begin{aligned}
H & =\left\{\boldsymbol{u}=\left(u_{i}\right) / u_{i} \in L^{2}(\Omega)\right\}, \quad \mathcal{H}=\left\{\boldsymbol{\sigma}=\left(\sigma_{i j}\right) / \sigma_{i j}=\sigma_{j i} \in L^{2}(\Omega)\right\}, \\
H_{1} & =\left\{\boldsymbol{u}=\left(u_{i}\right) / \boldsymbol{\varepsilon}(\boldsymbol{u}) \in \mathcal{H}\right\}, \quad \mathcal{H}_{1}=\{\boldsymbol{\sigma} \in \mathcal{H} / \text { Div } \boldsymbol{\sigma} \in H\} .
\end{aligned}
$$

Here $\varepsilon$ and Div are the deformation and divergence operators, respectively, defined by

$$
\boldsymbol{\varepsilon}(\boldsymbol{u})=\left(\varepsilon_{i j}(\boldsymbol{u})\right), \quad \varepsilon_{i j}(\boldsymbol{u})=\frac{1}{2}\left(u_{i, j}+u_{j, i}\right), \operatorname{Div} \boldsymbol{\sigma}=\left(\sigma_{i j, j}\right) .
$$

The spaces $H, \mathcal{H}, H_{1}$ and $\mathcal{H}_{1}$ are real Hilbert spaces endowed with the canonical inner products given by

$$
\begin{aligned}
(\boldsymbol{u}, \boldsymbol{v})_{H} & =\int_{\Omega} u_{i} v_{i} d x \quad \forall \boldsymbol{u}, \boldsymbol{v} \in H, \quad(\boldsymbol{\sigma}, \boldsymbol{\tau})_{\mathcal{H}}=\int_{\Omega} \sigma_{i j} \tau_{i j} d x \quad \forall \boldsymbol{\sigma}, \boldsymbol{\tau} \in \mathcal{H}, \\
(\boldsymbol{u}, \boldsymbol{v})_{H_{1}} & =(\boldsymbol{u}, \boldsymbol{v})_{H}+(\boldsymbol{\varepsilon}(\boldsymbol{u}), \boldsymbol{\varepsilon}(\boldsymbol{v}))_{\mathcal{H}} \quad \forall \boldsymbol{u}, \boldsymbol{v} \in H_{1} \\
(\boldsymbol{\sigma}, \boldsymbol{\tau})_{\mathcal{H}_{1}} & =(\boldsymbol{\sigma}, \boldsymbol{\tau})_{\mathcal{H}}+(\operatorname{Div} \boldsymbol{\sigma}, \operatorname{Div} \boldsymbol{\tau})_{H} \quad \forall \boldsymbol{\sigma}, \boldsymbol{\tau} \in \mathcal{H}_{1} .
\end{aligned}
$$

The associated norms on the spaces $H, \mathcal{H}, H_{1}$ and $\mathcal{H}_{1}$ are denoted by $\left.\left.\right|_{\cdot}\right|_{H}$, $|\cdot|_{\mathcal{H}},|\cdot|_{H_{1}}$ and $|\cdot|_{\mathcal{H}_{1}}$, respectively. For every element $\boldsymbol{v} \in H_{1}$ we also use the notation $\boldsymbol{v}$ for the trace of $\boldsymbol{v}$ on $\Gamma$ and we denote by $v_{\nu}$ and $\boldsymbol{v}_{\tau}$ the normal and the tangential components of $\boldsymbol{v}$ on $\Gamma$ given by $v_{\nu}=\boldsymbol{v} \cdot \boldsymbol{\nu}, \boldsymbol{v}_{\tau}=\boldsymbol{v}-v_{\nu} \boldsymbol{\nu}$. We also denote by $\sigma_{\nu}$ and $\boldsymbol{\sigma}_{\tau}$ the normal and the tangential traces of a function $\boldsymbol{\sigma} \in \mathcal{H}_{1}$, we recall that when $\boldsymbol{\sigma}$ is a regular function then $\sigma_{\nu}=(\boldsymbol{\sigma} \boldsymbol{\nu}) \cdot \boldsymbol{\nu}, \boldsymbol{\sigma}_{\tau}=\boldsymbol{\sigma} \boldsymbol{\nu}-\sigma_{\nu} \boldsymbol{\nu}$, and the following Green's formula holds

$$
(\boldsymbol{\sigma}, \boldsymbol{\varepsilon}(\boldsymbol{v}))_{\mathcal{H}}+(\operatorname{Div} \boldsymbol{\sigma}, \boldsymbol{v})_{H}=\int_{\Gamma} \boldsymbol{\sigma} \boldsymbol{\nu} \cdot \boldsymbol{\nu} d a \forall \boldsymbol{v} \in H_{1}
$$

Let $T>0$. For every real Banach space $X$ we use the classical notation for the spaces $C(0, T ; X), C^{1}(0, T ; X)$, and we use the standard notation for the Lebesgue spaces and for the Sobolev spaces $L^{p}(0, T ; X)$ and $W^{k, p}(0, T ; X)$, $1 \leq p \leq \infty, 1 \leq k$. Moreover, if $X_{1}$ and $X_{2}$ are real Hilbert spaces then $X_{1} \times X_{2}$ denotes the product. Hilbert space endowed with the canonical inner product $(., .)_{X_{1} \times X_{2}}$.

\section{Mechanical and variational formulations}

The physical setting is the following. An electro-viscoelastic body with long memory and damage occupies a bounded domain $\Omega \subset \mathbb{R}^{d}(d=2,3)$ with outer Lipschitz surface $\Gamma$. The body is submitted to the action of body forces of density $\boldsymbol{f}_{0}$ and volume electric charges of density $q_{0}$. It is also constrained mechanically and electrically on the boundary. We consider a partition of $\Gamma$ into three disjoint measurable parts $\Gamma_{1}, \Gamma_{2}$ and $\Gamma_{3}$, on one hand, and a partition of $\Gamma_{1} \cup \Gamma_{2}$ into two disjoint measurable parts $\Gamma_{a}$ and $\Gamma_{b}$, on the other hand, 
such that meas $\left(\Gamma_{1}\right)>0$, meas $\left(\Gamma_{a}\right)>0$. Let $T>0$ and let $[0, T]$ be the time interval of interest. The body is clamped on $\Gamma_{1}$, so the displacement field vanishes there. Surface tractions of density $\boldsymbol{f}_{2}$ act on $\Gamma_{2}$. We also assume that the electrical potential vanishes on $\Gamma_{a}$ and a surface free electrical charge of density $q_{2}$ is prescribed on $\Gamma_{b}$. In the reference configuration the body may come in contact over $\Gamma_{3}$ with a conductive moving obstacle, which is also called the foundation. The contact is modeled with the normal compliance condition and a general version of Coulomb's law of dry friction. Also, there may be electrical charges on the part of the body which is in contact with the foundation and which vanish when contact is lost. We are interested in the evolution of the deformation of the body and of the electric potential on the time interval $[0, T]$. The process is assumed to be isothermal, electrically static, i.e., all radiation effects are neglected, and mechanically quasistatic, i.e., the inertial terms in the momentum balance equations are neglected. To simplify the notation, we do not indicate explicitly the dependence of various functions on the variables $\mathbf{x} \in \Omega \cup \Gamma$ and $t \in[0, T]$. Then, the classical formulation of the mechanical problem of sliding frictional contact problem with normal compliance and wear may be stated as follows.

Problem P. Find a displacement field $\boldsymbol{u}: \Omega \times[0, T] \rightarrow \mathbb{R}^{d}$, a stress field $\boldsymbol{\sigma}: \Omega \times[0, T] \rightarrow \mathbb{S}^{d}$, an electric potential field $\varphi: \Omega \times[0, T] \rightarrow \mathbb{R}$, an electric displacement field $\boldsymbol{D}: \Omega \times[0, T] \rightarrow \mathbb{R}^{d}$, a damage field $\beta: \Omega \times[0, T] \rightarrow \mathbb{R}$ and a wear function $\zeta: \Gamma_{3} \times[0, T] \rightarrow \mathbb{R}$ such that

$$
\begin{aligned}
& \boldsymbol{\sigma}=\mathcal{A} \varepsilon(\dot{\boldsymbol{u}})+\mathcal{F}(\boldsymbol{\varepsilon}(\boldsymbol{u}), \beta)+\int_{0}^{t} M(t-s) \boldsymbol{\varepsilon}(\boldsymbol{u}(s)) d s+\mathcal{E}^{*} \nabla \varphi \text { in } \Omega \times(0, T), \\
& \boldsymbol{D}=\mathcal{E} \boldsymbol{\varepsilon}(\boldsymbol{u})-\boldsymbol{B} \nabla \varphi \quad \text { in } \Omega \times(0, T), \\
& \dot{\beta}-k_{e} \triangle \beta+\partial \Psi_{K}(\beta) \ni S(\varepsilon(\boldsymbol{u}), \beta) \quad \text { in } \Omega \times(0, T), \\
& \text { Div } \boldsymbol{\sigma}+\boldsymbol{f}_{0}=\mathbf{0} \quad \text { in } \Omega \times(0, T) \text {, } \\
& \operatorname{div} \boldsymbol{D}=q_{0} \quad \text { in } \Omega \times(0, T), \\
& \boldsymbol{u}=\mathbf{0} \quad \text { on } \quad \Gamma_{1} \times(0, T), \quad \boldsymbol{\sigma} \boldsymbol{\nu}=\boldsymbol{f}_{2} \quad \text { on } \quad \Gamma_{2} \times(0, T), \\
& \left\{\begin{array}{l}
-\sigma_{\nu}=p_{\nu}, \\
\left|\boldsymbol{\sigma}_{\tau}\right| \leq \mu p_{\nu}, \\
\boldsymbol{\sigma}_{\tau}=-\mu p_{\nu} \frac{\left(\dot{\boldsymbol{u}}_{\tau}-\boldsymbol{v}^{*}\right)}{\left|\dot{\boldsymbol{u}}_{\tau}-\boldsymbol{v}^{*}\right|} \quad \text { if } \quad \dot{\boldsymbol{u}}_{\tau} \neq \boldsymbol{v}^{*}, \\
\dot{\zeta}=k_{1} \mu p_{\nu} R^{*}\left(\left|\dot{\boldsymbol{u}}_{\tau}-\boldsymbol{v}^{*}\right|\right), \quad \text { on } \Gamma_{3} \times(0, T),
\end{array}\right. \\
& \frac{\partial \beta}{\partial \boldsymbol{\nu}}=0 \quad \text { on } \Gamma \times(0, T) \text {, } \\
& \varphi=0 \quad \text { on } \quad \Gamma_{a} \times(0, T), \quad \boldsymbol{D} \cdot \boldsymbol{\nu}=q_{2} \quad \text { on } \quad \Gamma_{b} \times(0, T), \\
& \boldsymbol{D} \cdot \boldsymbol{\nu}=\psi\left(u_{\nu}-h-\zeta\right) \phi_{L}\left(\varphi-\varphi_{0}\right) \quad \text { on } \Gamma_{3} \times(0, T) \text {, } \\
& \boldsymbol{u}(0)=\boldsymbol{u}_{0}, \beta(0)=\beta_{0}, \zeta(0)=0 \quad \text { in } \quad \Omega \text {. }
\end{aligned}
$$

Here, $\mu=\mu\left(\zeta,\left|\dot{\boldsymbol{u}}_{\tau}-\boldsymbol{v}^{*}\right|\right)$ and $p_{\nu}=p_{\nu}\left(u_{\nu}-h-\zeta\right)$. Equations (3.1) and (3.2) represent the constitutive law for a piezoelectric material with long memory and damage, where $\mathcal{A}$ and $\mathcal{F}$ are nonlinear operators describing the purely viscous and the elastic properties of the material, respectively, $M$ is a relaxation 
fourth order tensor, $\boldsymbol{E}(\varphi)=-\nabla \varphi$ is the electric field, $\mathcal{E}=\left(e_{i j k}\right)$ represents the third order piezoelectric tensor, $\mathcal{E}^{*}$ is its transposed and $\boldsymbol{B}$ denotes the electric permittivity tensor. We use dots for derivatives with respect to the time variable $t$. Inclusion (3.3) describes the evolution of the damage field, governed by the source damage function $S$, where $k_{e}$ is a positive coefficient and $\partial \Psi_{K}$ is the subdifferential of indicator function of the set of admissible damage functions given by $K=\left\{\xi \in H^{1}(\Omega) / 0 \leq \xi \leq 1\right.$ a.e. in $\left.\Omega\right\}$. Equations (3.4) and (3.5) represent the equilibrium equations for the stress and electric displacement fields. Equations (3.6) are the displacement and traction boundary conditions, respectively. Equation (3.8) describes a homogeneous Neumann boundary condition, where $\frac{\partial \beta}{\partial \nu}$ is the normal derivative of $\beta$. Equations (3.9) represent the electric boundary conditions. In (3.11) $\boldsymbol{u}_{0}$ is the given initial displacement, $\beta_{0}$ is the initial material damage and $\zeta(0)=0$ means that at the initial moment the body is not subject to any prior wear. The relations (3.7) represent the condition with normal compliance, friction and wear. The wear function $\zeta$ which measures the wear accumulated of the surface. The evolution of the wear of the contacting surface is governed by the differential form of Archard's law ( see, e.g., $[1,19,28,29])$, where $k_{1}>0$ is a wear coefficient, $p_{\nu}$ is a prescribed function of the normal compliance, $\mu \geq 0$ is the coefficient of friction, $h$ represents the gap in direction of $\boldsymbol{\nu}, \boldsymbol{v}^{*}$ is the tangential velocity of the foundation, $\left|\dot{\boldsymbol{u}}_{\tau}-\boldsymbol{v}^{*}\right|$ represents the slip rate between the contact surface and the foundation, and $R^{*}: \mathbb{R}_{+} \rightarrow \mathbb{R}_{+}$is the truncation operator $R^{*}(r)=r$ if $r \leq R$ and $R^{*}(r)=R$ if $r>R, R$ being a fixed positive constant. We recall that in the case without wear, a general version of normal compliance is given by $-\sigma_{\nu}=p_{\nu}\left(u_{\nu}-h\right)$. The difference $u_{\nu}-h$, when positive, represents the penetration of the surface asperities into those of the foundation. This condition was first introduced in [11] and used in a large number of papers, see for instance $[6,13]$ and the references therein. Next, (3.10) is the electrical contact condition on $\Gamma_{3}$, which is the main novelty of this work. This condition has been used in [9] for electroviscoelastic contact problem with short memory. It represents a regularized condition which may be obtained as follows. First, we assume that the foundation is electrically conductive and its potential is maintained at $\varphi_{0}$. When there is no contact at a point on the surface (i.e., $u_{\nu}<h$ ), there are no free electrical charges on the surface and the normal component of the electric displacement field vanishes (i.e., $\boldsymbol{D} \cdot \boldsymbol{\nu}=0$ ). During the process of contact (i.e., when $u_{\nu} \geq h$ ) the normal component of the electric displacement field or the free charge is assumed to be proportional to the difference between the potential of the foundation and the body's surface potential with $k$ as the proportionality factor. Thus $\boldsymbol{D} \cdot \boldsymbol{\nu}=k\left(\varphi-\varphi_{0}\right)$, then $\boldsymbol{D} \cdot \boldsymbol{\nu}=k \chi_{[0, \infty)}\left(u_{\nu}-h\right)\left(\varphi-\varphi_{0}\right)$, where $\chi_{[0, \infty)}$ is the characteristic function of the interval $[0, \infty)$. Since our process involves the wear of the contacting surfaces we need to take into account the change in the geometry by replacing the initial gap function $h$ with $h+\zeta$ during the process, we write $\boldsymbol{D} \cdot \boldsymbol{\nu}=k \chi_{[0, \infty)}\left(u_{\nu}-h-\zeta\right)\left(\varphi-\varphi_{0}\right)$, this condition describes perfect electrical contact and is somewhat similar to the well-known Signorini contact condition. Both conditions may be over-idealizations in many applications. To make it more realistic, we regularize previous relation and write it as (3.10) in which $k \chi_{[0, \infty)}\left(u_{\nu}-h-\zeta\right)$ is replaced with $\psi$ which is a regular function 
which will be described below, and $\phi_{L}$ is the truncation function $\phi_{L}(s)=-L$ if $s<-L, \phi_{L}(s)=s$ if $-L \leq s \leq L$ and $\phi_{L}(s)=L$ if $s>L$, where $L$ is a large positive constant. We note that this truncation does not pose any practical limitations on the applicability of the model, since $L$ may be arbitrarily large, higher than any possible peak voltage in the system, and therefore in applications $\phi_{L}\left(\varphi-\varphi_{0}\right)=\varphi-\varphi_{0}$.

To obtain a variational formulation of the problem (3.1)-(3.11) we introduce the closed subspace of $H_{1}$ defined by $V=\left\{\boldsymbol{v} \in H_{1} / \boldsymbol{v}=\mathbf{0}\right.$ on $\left.\Gamma_{1}\right\}$. Since meas $\left(\Gamma_{1}\right)>0$, Korn's inequality holds and there exists a constant $c_{K}>0$ which depends only on $\Omega$ and $\Gamma_{1}$ such that

$$
|\varepsilon(\boldsymbol{v})|_{\mathcal{H}} \geq c_{K}|\boldsymbol{v}|_{H_{1}} \forall \boldsymbol{v} \in V
$$

On the space $V$ we consider the inner product and the associated norm given by

$$
(\boldsymbol{u}, \boldsymbol{v})_{V}=(\varepsilon(\boldsymbol{u}), \boldsymbol{\varepsilon}(\boldsymbol{v}))_{\mathcal{H}}, \quad|\boldsymbol{v}|_{V}=|\varepsilon(\boldsymbol{v})|_{\mathcal{H}} \forall \boldsymbol{v} \in V .
$$

It follows from Korn's inequality that $|\cdot|_{H_{1}}$ and $|\cdot|_{V}$ are equivalent norms on $V$. Therefore $\left(V,|\cdot|_{V}\right)$ is a real Hilbert space. Moreover, by the Sobolev's trace theorem and Korn's inequality, there exists a constant $c_{0}>0$, depending only on $\Omega, \Gamma_{1}$ and $\Gamma_{3}$ such that

$$
|\boldsymbol{v}|_{L^{2}\left(\Gamma_{3}\right)^{d}} \leq c_{0}|\boldsymbol{v}|_{V} \quad \forall \boldsymbol{v} \in V
$$

We also introduce the spaces

$$
\begin{aligned}
& W=\left\{\phi \in H^{1}(\Omega) / \phi=0 \text { on } \Gamma_{a}\right\}, \\
& \mathcal{W}=\left\{\boldsymbol{D}=\left(D_{i}\right) / D_{i} \in L^{2}(\Omega), \operatorname{div} \boldsymbol{D} \in L^{2}(\Omega)\right\},
\end{aligned}
$$

where $\operatorname{div} \boldsymbol{D}=\left(D_{i, i}\right)$. The spaces $W$ and $\mathcal{W}$ are real Hilbert spaces with the inner products given by

$$
(\varphi, \phi)_{W}=\int_{\Omega} \nabla \varphi \cdot \nabla \phi d x, \quad(\boldsymbol{D}, \boldsymbol{E})_{\mathcal{W}}=\int_{\Omega} \boldsymbol{D} \cdot \boldsymbol{E} d x+\int_{\Omega} \operatorname{div} \boldsymbol{D} \cdot \operatorname{div} \boldsymbol{E} d x .
$$

The associated norms will be denoted by $|\cdot|_{W}$ and $|\cdot|_{\mathcal{W}}$, respectively. Moreover, when $\boldsymbol{D} \in \mathcal{W}$ is a regular function, the following Green's type formula holds

$$
(\boldsymbol{D}, \nabla \phi)_{H}+(\operatorname{div} \boldsymbol{D}, \phi)_{L^{2}(\Omega)}=\int_{\Gamma} \boldsymbol{D} \cdot \boldsymbol{\nu} \phi d a \quad \forall \phi \in H^{1}(\Omega) .
$$

Notice also that, since meas $\left(\Gamma_{a}\right)>0$, the following Friedrichs-Poincaré inequality holds

$$
|\nabla \phi|_{H} \geq c_{F}|\phi|_{H^{1}(\Omega)} \forall \phi \in W
$$

where $c_{F}>0$ is a constant which depends only on $\Omega$ and $\Gamma_{a}$. It follows from Friedrichs-Poincaré inequality that $|\cdot|_{H^{1}(\Omega)}$ and $|\cdot|_{W}$ are equivalent norms on $W$ and therefore $\left(W,|.|_{W}\right)$ is a real Hilbert space. Moreover, by the Sobolev's trace theorem, there exists a constant $a_{0}>0$, depending only on $\Omega, \Gamma_{a}$ and $\Gamma_{3}$ such that

$$
|\phi|_{L^{2}\left(\Gamma_{3}\right)} \leq a_{0}|\phi|_{W} \quad \forall \phi \in W
$$


In the study of the mechanical problem (3.1)-(3.11), we now list assumptions on the data. Assume that the operators $\mathcal{A}, \mathcal{F}, \mathcal{E}, \boldsymbol{B}$ and the functions $S, p_{\nu}, \mu$, $\psi$ satisfy the following conditions with $L_{\mathcal{A}}, m_{\mathcal{A}}, L_{\mathcal{F}}, L_{S}, L_{\nu}, p_{\nu}^{*}, L_{\mu}, \mu^{*}, m_{B}$, $L_{\psi}$ and $N_{\psi}$ being positive constants:

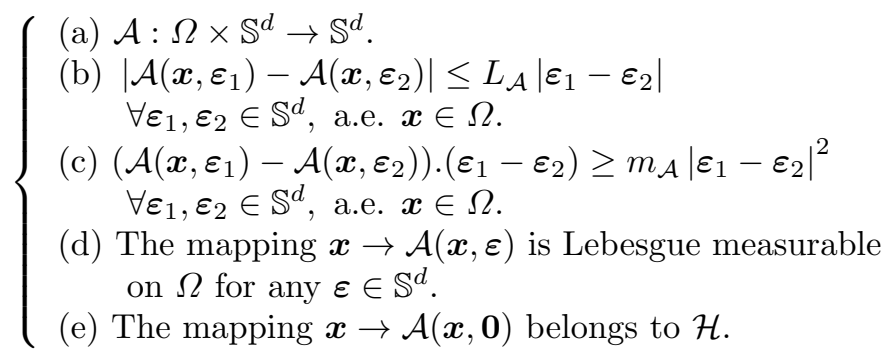

(a) $\mathcal{F}: \Omega \times \mathbb{S}^{d} \times \mathbb{R} \rightarrow \mathbb{S}^{d}$.

(b) $\left|\mathcal{F}\left(\boldsymbol{x}, \varepsilon_{1}, r_{1}\right)-\mathcal{F}\left(\boldsymbol{x}, \varepsilon_{2}, r_{2}\right)\right| \leq L_{\mathcal{F}}\left(\left|\varepsilon_{1}-\varepsilon_{2}\right|+\left|r_{1}-r_{2}\right|\right)$ $\forall \varepsilon_{1}, \varepsilon_{2} \in \mathbb{S}^{d}, \forall r_{1}, r_{2} \in \mathbb{R}$ a.e. $\boldsymbol{x} \in \Omega$.

(c) The mapping $\boldsymbol{x} \rightarrow \mathcal{F}(\boldsymbol{x}, \boldsymbol{\varepsilon}, r)$ is Lebesgue measurable on $\Omega$ for any $\varepsilon \in \mathbb{S}^{d}$ and $r \in \mathbb{R}$.

(d) The mapping $\boldsymbol{x} \rightarrow \mathcal{F}(\boldsymbol{x}, \mathbf{0}, 0)$ belongs to $\mathcal{H}$.

(a) $S: \Omega \times \mathbb{S}^{d} \times \mathbb{R} \rightarrow \mathbb{R}$.

(b) $\left|S\left(\boldsymbol{x}, \boldsymbol{\varepsilon}_{1}, r_{1}\right)-S\left(\boldsymbol{x}, \boldsymbol{\varepsilon}_{2}, r_{2}\right)\right| \leq L_{S}\left(\left|\varepsilon_{1}-\varepsilon_{2}\right|+\left|r_{1}-r_{2}\right|\right)$ $\forall \varepsilon_{1}, \varepsilon_{2} \in \mathbb{S}^{d}, \forall r_{1}, r_{2} \in \mathbb{R}$ a.e. $\boldsymbol{x} \in \Omega$.

(c) For any $\varepsilon \in \mathbb{S}^{d}$ and $r \in \mathbb{R}$, $\boldsymbol{x} \rightarrow S(\boldsymbol{x}, \boldsymbol{\varepsilon}, r)$ is Lebesgue measurable on $\Omega$.

(d) The mapping $\boldsymbol{x} \rightarrow S(\boldsymbol{x}, \mathbf{0}, 0)$ belongs to $L^{2}(\Omega)$.

(a) $p_{\nu}: \Gamma_{3} \times \mathbb{R} \rightarrow \mathbb{R}_{+}$.

(b) $\left|p_{\nu}\left(\boldsymbol{x}, u_{1}\right)-p_{\nu}\left(\boldsymbol{x}, u_{2}\right)\right| \leq L_{\nu}\left|u_{1}-u_{2}\right|$

$\forall u_{1}, u_{2} \in \mathbb{R}$, a.e. $\boldsymbol{x} \in \Gamma_{3}$.

(c) For any $u \in \mathbb{R} \boldsymbol{x} \rightarrow p_{\nu}(\boldsymbol{x}, u)$ is Lebesgue measurable on $\Gamma_{3}$.

(d) $p_{\nu}(\boldsymbol{x}, u)=0$ for all $u \leq 0$, a.e. $\boldsymbol{x} \in \Gamma_{3}$.

(e) $p_{\nu}(\boldsymbol{x}, u) \leq p_{\nu}^{*}$ for all $u \in \mathbb{R}$, a.e. $\boldsymbol{x} \in \Gamma_{3}$.
(a) $\mu: \Gamma_{3} \times \mathbb{R}^{2} \rightarrow \mathbb{R}$.
(b) $\left|\mu\left(\boldsymbol{x}, a_{1}, b_{1}\right)-\mu\left(\boldsymbol{x}, a_{2}, b_{2}\right)\right| \leq L_{\mu}\left(\left|a_{1}-a_{2}\right|+\left|b_{1}-b_{2}\right|\right)$ $\forall a_{1}, a_{2}, b_{1}, b_{2} \in \mathbb{R}$, a.e. $\boldsymbol{x} \in \Gamma_{3}$. (c) The mapping $\boldsymbol{x} \rightarrow \mu(\boldsymbol{x}, a, b)$ is Lebesgue
measurable on $\Gamma_{3} \forall a, b \in \mathbb{R}$.
(d) $\mu(\boldsymbol{x}, a, b) \leq \mu^{*} \forall a, b \in \mathbb{R}$.a.e. $\boldsymbol{x} \in \Gamma_{3}$.
$\int\left(\right.$ a) $\boldsymbol{B}=\left(B_{i j}\right): \Omega \times \mathbb{R}^{d} \rightarrow \mathbb{R}^{d}$.
$\left\{\right.$ (b) $\boldsymbol{B}(\boldsymbol{x}) \boldsymbol{E}=\left(b_{i j}(\boldsymbol{x}) E_{j}\right) \forall \boldsymbol{E}=\left(E_{i}\right) \in \mathbb{R}^{d}$, a.e. $\boldsymbol{x} \in \Omega$.
(c) $b_{i j}=b_{j i}, \quad b_{i j} \in L^{\infty}(\Omega)$.
(d) $\boldsymbol{B} \boldsymbol{E} \cdot \boldsymbol{E} \geq m_{B}|\boldsymbol{E}|^{2} \quad \forall \boldsymbol{E}=\left(E_{i}\right) \in \mathbb{R}^{d}$, a.e. $\boldsymbol{x} \in \Omega$.
$\int\left(\right.$ a) $\mathcal{E}: \Omega \times \mathbb{S}^{d} \rightarrow \mathbb{R}^{d}$.
(b) $\mathcal{E}(\boldsymbol{x}) \boldsymbol{\tau}=\left(e_{i j k}(\boldsymbol{x}) \tau_{j k}\right) \forall \boldsymbol{\tau}=\left(\tau_{i j}\right) \in \mathbb{S}^{d}$, a.e. $\boldsymbol{x} \in \Omega$.
(c) $e_{i j k}=e_{i k j} \in L^{\infty}(\Omega)$.




$$
\left\{\begin{array}{l}
\text { (a) } \psi: \Gamma_{3} \times \mathbb{R} \rightarrow \mathbb{R}_{+} \cdot \\
\text { (b) }\left|\psi\left(\boldsymbol{x}, u_{1}\right)-\psi\left(\boldsymbol{x}, u_{2}\right)\right| \leq L_{\psi}\left|u_{1}-u_{2}\right| \\
\forall u_{1}, u_{2} \in \mathbb{R}, \text { a.e. } \boldsymbol{x} \in \Gamma_{3} . \\
\text { (c) }|\psi(\boldsymbol{x}, u)| \leq N_{\psi} \forall u \in \mathbb{R} \text {, a.e. } \boldsymbol{x} \in \Gamma_{3} . \\
\text { (d) } \boldsymbol{x} \rightarrow \psi(\boldsymbol{x}, u) \text { is measurable on } \Gamma_{3} \text { for all } u \in \mathbb{R} . \\
\text { (e) } \boldsymbol{x} \rightarrow \psi(\boldsymbol{x}, u)=0 \text { for all } u \leq 0 .
\end{array}\right.
$$

The relaxation tensor $M$ satisfies $M \in C\left(0, T ; \mathcal{H}_{\infty}\right)$, where $\mathcal{H}_{\infty}$ is the space of fourth order tensor field given by

$$
\mathcal{H}_{\infty}=\left\{E=\left(E_{i j k l}\right) / E_{i j k l}=E_{k l i j}=E_{j i k l} \in L^{\infty}(\Omega), 1 \leq i, j, k, l \leq d\right\},
$$

which is a real Banach space with the norm

$$
|E|_{\mathcal{H}_{\infty}}=\max _{1 \leq i, j, k, l \leq d}\left|E_{i j k l}\right|_{L^{\infty}(\Omega)} .
$$

The density of volume forces, traction, volume electric charges and surface electric charges have the regularity

$$
\begin{aligned}
& \boldsymbol{f}_{0} \in C(0, T ; H), \quad \boldsymbol{f}_{2} \in C\left(0, T ; L^{2}\left(\Gamma_{2}\right)^{d}\right) . \\
& q_{0} \in C\left(0, T ; L^{2}(\Omega)\right), q_{2} \in C\left(0, T ; L^{2}\left(\Gamma_{b}\right)\right) .
\end{aligned}
$$

Finally, we assume that the gap function $h$, the given potential of the foundation $\varphi_{0}$, the initial displacement field $\boldsymbol{u}_{0}$ and the initial damage field $\beta_{0}$ satisfy

$$
h \in L^{2}\left(\Gamma_{3}\right), h \geq 0 \text { a.e. } \boldsymbol{x} \in \Gamma_{3}, \quad \varphi_{0} \in L^{2}\left(\Gamma_{3}\right), \boldsymbol{u}_{0} \in V, \beta_{0} \in K .
$$

We define the bilinear form $a: H^{1}(\Omega) \times H^{1}(\Omega) \rightarrow \mathbb{R}$ by

$$
a(\varsigma, \vartheta)=k_{e} \int_{\Omega} \nabla \varsigma \cdot \nabla \vartheta d x
$$

Next we define the four mappings $f:[0, T] \rightarrow V, q:[0, T] \rightarrow W, j: V \times V \times$ $V \times L^{2}\left(\Gamma_{3}\right) \rightarrow \mathbb{R}$ and $\gamma: V \times W \times L^{2}\left(\Gamma_{3}\right) \rightarrow W$, respectively, by

$$
\begin{aligned}
& (\boldsymbol{f}(t), \boldsymbol{v})_{V}=\int_{\Omega} \boldsymbol{f}_{0}(t) \cdot \boldsymbol{v} d x+\int_{\Gamma_{2}} \boldsymbol{f}_{2}(t) \cdot \boldsymbol{v} d a \\
& (q(t), \phi)_{W}=\int_{\Omega} q_{0}(t) \phi d x-\int_{\Gamma_{b}} q_{2}(t) \phi d a \\
& \quad j(\boldsymbol{u}, \boldsymbol{v}, \boldsymbol{w}, \zeta)=\int_{\Gamma_{3}} p_{\nu}\left(u_{\nu}-h-\zeta\right) w_{\nu} d a \\
& +\int_{\Gamma_{3}} \mu p_{\nu}\left(u_{\nu}-h-\zeta\right)\left|\boldsymbol{w}_{\tau}-\boldsymbol{v}^{*}\right| d a \\
& (\gamma(\boldsymbol{u}, \varphi, \zeta), \phi)_{W}=\int_{\Gamma_{3}} \psi\left(u_{\nu}-h-\zeta\right) \phi_{L}\left(\varphi-\varphi_{0}\right) \phi d a,
\end{aligned}
$$

for all $\boldsymbol{u}, \boldsymbol{v}, \boldsymbol{w} \in V, \zeta \in L^{2}\left(\Gamma_{3}\right), \varphi, \phi \in W$ and $t \in[0, T], \mu=\mu\left(\zeta,\left|\boldsymbol{v}_{\tau}-\boldsymbol{v}^{*}\right|\right)$. 
The functional $j: V \times V \times V \times L^{2}\left(\Gamma_{3}\right) \rightarrow \mathbb{R}$ satisfies

$$
\left\{\begin{array}{l}
\text { for all } \boldsymbol{g}, \boldsymbol{w} \in V \text { and } \zeta \in L^{2}\left(\Gamma_{3}\right): \boldsymbol{v} \rightarrow j(\boldsymbol{g}, \boldsymbol{w}, \boldsymbol{v}, \zeta) \\
\text { is proper, convex and lower semicontinuous on } V .
\end{array}\right.
$$

We note that conditions (3.19) and (3.20) imply that

$$
\boldsymbol{f} \in C(0, T ; V), q \in C(0, T ; W) .
$$

Using standard arguments we obtain the variational formulation of the mechanical problem (3.1)-(3.11).

Problem PV. Find a displacement field $\boldsymbol{u}:[0, T] \rightarrow V$, a stress field $\boldsymbol{\sigma}$ : $[0, T] \rightarrow \mathcal{H}_{1}$, an electric potential field $\varphi:[0, T] \rightarrow W$, an electric displacement field $\boldsymbol{D}:[0, T] \rightarrow \mathcal{W}$, a damage field $\beta:[0, T] \rightarrow H^{1}(\Omega)$ and a wear function $\zeta:[0, T] \rightarrow L^{2}\left(\Gamma_{3}\right)$ such that,

$$
\begin{aligned}
& \boldsymbol{\sigma}(t)=\mathcal{A} \varepsilon(\dot{\boldsymbol{u}}(t))+\mathcal{F}(\varepsilon(\boldsymbol{u}(t)), \beta(t)) \\
& +\mathcal{E}^{*} \nabla \varphi(t)+\int_{0}^{t} M(t-s) \varepsilon(\boldsymbol{u}(s)) d s, t \in[0, T], \\
& (\boldsymbol{\sigma}(t), \boldsymbol{\varepsilon}(\boldsymbol{v}-\dot{\boldsymbol{u}}(t)))_{\mathcal{H}}+j(\boldsymbol{u}(t), \dot{\boldsymbol{u}}(t), \boldsymbol{v}, \zeta(t)) \\
& -j(\boldsymbol{u}(t), \dot{\boldsymbol{u}}(t), \dot{\boldsymbol{u}}(t), \zeta(t)) \geq(\boldsymbol{f}(t), \boldsymbol{v}-\dot{\boldsymbol{u}}(t))_{V} \forall \boldsymbol{v} \in V, t \in[0, T], \\
& \beta(t) \in K, \quad(\dot{\beta}(t), \xi-\beta(t))_{L^{2}(\Omega)}+a(\beta(t), \xi-\beta(t)) \\
& \geq(S(\varepsilon(\boldsymbol{u}(t)), \beta(t)), \xi-\beta(t))_{L^{2}(\Omega)} \quad \forall \xi \in K \text {, a.e. } t \in(0, T), \\
& \boldsymbol{D}(t)=\mathcal{E} \boldsymbol{\varepsilon}(\boldsymbol{u}(t))-\boldsymbol{B} \nabla \varphi(t), t \in[0, T], \\
& (\boldsymbol{D}(t), \nabla \phi)_{H}=(\gamma(\boldsymbol{u}(t), \varphi(t), \zeta(t)), \phi)_{W} \\
& -(q(t), \phi)_{W} \quad \forall \phi \in W, t \in[0, T] \text {, } \\
& \dot{\zeta}=k_{1} \mu\left(\zeta,\left|\dot{\boldsymbol{u}}_{\tau}-\boldsymbol{v}^{*}\right|\right) p_{\nu}\left(u_{\nu}-h-\zeta\right) R^{*}\left(\left|\dot{\boldsymbol{u}}_{\tau}-\boldsymbol{v}^{*}\right|\right), t \in[0, T], \\
& \boldsymbol{u}(0)=\boldsymbol{u}_{0}, \beta(0)=\beta_{0}, \zeta(0)=0 \text {. }
\end{aligned}
$$

The existence of the unique solution to Problem PV is stated and proved in the next section.

\section{Existence and uniqueness result}

The main result in this section is the following existence and uniqueness result.

Theorem 1. Assume that (3.13)-(3.21) and (3.25) hold. Then if $c_{0}^{2} p_{\nu}^{*} L_{\mu}<$ $m_{\mathcal{A}}$ and $N_{\psi}<\frac{m_{B}}{a_{0}^{2}}$, there exists a unique solution $\{\boldsymbol{u}, \boldsymbol{\sigma}, \boldsymbol{\varphi}, \boldsymbol{D}, \beta, \zeta\}$ to Problem $P V$. Moreover, the solution satisfies

$$
\begin{aligned}
& \boldsymbol{u} \in C^{1}(0, T ; V), \\
& \boldsymbol{\sigma} \in C\left(0, T ; \mathcal{H}_{1}\right), \\
& \varphi \in C(0, T ; W), \quad \boldsymbol{D} \in C(0, T ; \mathcal{W}), \\
& \beta \in H^{1}\left(0, T ; L^{2}(\Omega)\right) \cap L^{2}\left(0, T ; H^{1}(\Omega)\right), \\
& \zeta \in C^{1}\left(0, T ; L^{2}\left(\Gamma_{3}\right)\right) .
\end{aligned}
$$


The functions $\boldsymbol{u}, \boldsymbol{\sigma}, \boldsymbol{\varphi}, \boldsymbol{D}, \beta$ and $\zeta$ which satisfy (3.26)-(3.28) are called weak solution to contact problem $\mathrm{P}$. We conclude that, under the assumptions (3.13)(3.21) and if $c_{0}^{2} p_{\nu}^{*} L_{\mu}<m_{\mathcal{A}}$ and $N_{\psi}<\frac{m_{B}}{a_{0}^{2}}$, the mechanical problem (3.1)-(3.11) has a unique weak solution satisfying (4.1)-(4.4). The proof of Theorem 1 is carried out in several steps. Everywhere in this section we suppose that assumptions of Theorem 1 hold. Below, $c$ denotes a generic positive constant which may depend on $\Omega, \Gamma_{1}, \Gamma_{2}, \Gamma_{3}, \mathcal{A}, \mathcal{E}, \mathcal{F}, M, \boldsymbol{B}, \psi, p_{\nu}, \mu$ and $T$ but does not depend on $t$ nor of the rest of input data, and whose value may change from place to place.

In the first step let $\zeta \in C\left(0, T ; L^{2}\left(\Gamma_{3}\right)\right), \boldsymbol{\eta} \in C(0, T ; \mathcal{H}), \boldsymbol{g} \in C(0, T ; V)$ and $\boldsymbol{w} \in C(0, T ; V)$ be given and consider the following variational problem.

Problem $\mathbf{P} \mathbf{V}_{\zeta \eta g w}$. Find a velocity field $\boldsymbol{v}_{\zeta \eta g w}:[0, T] \rightarrow V$ and a stress field $\boldsymbol{\sigma}_{\zeta \eta g w}:[0, T] \rightarrow \mathcal{H}$ such that for all $t \in[0, T]$,

$$
\begin{aligned}
& \boldsymbol{\sigma}_{\zeta \eta g w}(t)=\mathcal{A} \boldsymbol{\varepsilon}\left(\boldsymbol{v}_{\zeta \eta g w}(t)\right)+\boldsymbol{\eta}(t), \\
& \left.\left(\boldsymbol{\sigma}_{\zeta \eta g w}(t)\right), \boldsymbol{\varepsilon}\left(\boldsymbol{v}-\boldsymbol{v}_{\zeta \eta g w}(t)\right)\right)_{\mathcal{H}}+j(\boldsymbol{g}(t), \boldsymbol{w}(t), \boldsymbol{v}, \zeta(t)) \\
& \quad-j\left(\boldsymbol{g}(t), \boldsymbol{w}(t), \boldsymbol{v}_{\zeta \eta g w}(t), \zeta(t)\right) \geq\left(\boldsymbol{f}(t), \boldsymbol{v}-\boldsymbol{v}_{\zeta \eta g w}(t)\right)_{V} \forall \boldsymbol{v} \in V .
\end{aligned}
$$

In the study of Problem $\mathrm{PV}_{\zeta \eta g w}$ we have the following result.

Lemma 1. $P V_{\zeta \eta g w}$ has a unique solution $\left(\boldsymbol{v}_{\zeta \eta g w}, \boldsymbol{\sigma}_{\zeta \eta g w}\right)$ such that $\boldsymbol{v}_{\zeta \eta g w} \in C(0, T ; V), \boldsymbol{\sigma}_{\zeta \eta g w} \in C\left(0, T ; \mathcal{H}_{1}\right)$.

Proof. We define the operator $A: V \rightarrow V$ such that

$$
(A \boldsymbol{u}, \boldsymbol{v})_{V}=(\mathcal{A} \varepsilon(\boldsymbol{u}), \boldsymbol{\varepsilon}(\boldsymbol{v}))_{\mathcal{H}} \forall \boldsymbol{u}, \boldsymbol{v} \in V .
$$

It follows from (4.7), (3.13)(b) and (3.13)(c) that $A: V \rightarrow V$ is Lipschitz continuous and a strongly monotone operator on $V$. Moreover using Riesz Representation Theorem we may define an element $\boldsymbol{F} \in C(0, T ; V)$ by $(\boldsymbol{F}(t), \boldsymbol{v})_{V}=$ $(\boldsymbol{f}(t), \boldsymbol{v})_{V}-(\boldsymbol{\eta}(t), \boldsymbol{\varepsilon}(\boldsymbol{v}))_{\mathcal{H}}$. Since $A$ is a strongly monotone and Lipschitz continuous operator on $V$ and from (3.25), it follows from classical result on elliptic inequalities (see for example [5]) that there exists a unique function $\boldsymbol{v}_{\zeta \eta g w} \in V$ which satisfies

$$
\begin{aligned}
& \left(A \boldsymbol{v}_{\zeta \eta g w}(t), \boldsymbol{v}-\boldsymbol{v}_{\zeta \eta g w}(t)\right)_{V}+j(\boldsymbol{g}(t), \boldsymbol{w}(t), \boldsymbol{v}, \zeta(t)) \\
& \quad-j\left(\boldsymbol{g}(t), \boldsymbol{w}(t), \boldsymbol{v}_{\zeta \eta g w}(t), \zeta(t)\right) \geqslant\left(\boldsymbol{F}(t), \boldsymbol{v}-\boldsymbol{v}_{\zeta \eta g w}(t)\right)_{V} .
\end{aligned}
$$

We use the relation (4.5), the assumption (3.13) and the properties of the deformation tensor to obtain that $\boldsymbol{\sigma}_{\zeta \eta g w}(t) \in \mathcal{H}$. Since $\boldsymbol{v}=\boldsymbol{v}_{\zeta \eta g w}(t) \pm \Phi$ satisfies (4.6), where $\Phi \in \mathcal{D}(\Omega)^{d}$ is arbitrary, using the definition (3.22) we find

$$
\operatorname{Div}_{\zeta \eta g w}(t)+\boldsymbol{f}_{0}(t)=\mathbf{0} .
$$

With the regularity assumption (3.19) on $\boldsymbol{f}_{0}$ we see that Div $\boldsymbol{\sigma}_{\zeta \eta g w}(t) \in H$. Therefore $\boldsymbol{\sigma}_{\zeta \eta g w}(t) \in \mathcal{H}_{1}$. Next we show that $\boldsymbol{v}_{\zeta \eta g w} \in C(0, T ; V)$. Let $t_{1}, t_{2} \in$ $[0, T]$ and denote $\boldsymbol{v}_{\zeta \eta g w}\left(t_{i}\right)=\boldsymbol{v}_{i}, \boldsymbol{g}\left(t_{i}\right)=\boldsymbol{g}_{i}, \boldsymbol{w}\left(t_{i}\right)=\boldsymbol{w}_{i}, \zeta\left(t_{i}\right)=\zeta_{i}, \boldsymbol{\eta}\left(t_{i}\right)=\boldsymbol{\eta}_{i}$, $\boldsymbol{\sigma}\left(t_{i}\right)=\boldsymbol{\sigma}_{i}$ and $\boldsymbol{f}\left(t_{i}\right)=\boldsymbol{f}_{i}$ for $i=1,2$. Using the relation (4.8) we find

$$
\begin{aligned}
& \left(A \boldsymbol{v}_{1}-A \boldsymbol{v}_{2}, \boldsymbol{v}_{1}-\boldsymbol{v}_{2}\right)_{V} \leq j\left(\boldsymbol{g}_{1}, \boldsymbol{w}_{1}, \boldsymbol{v}_{2}, \zeta_{1}\right)-j\left(\boldsymbol{g}_{1}, \boldsymbol{w}_{1}, \boldsymbol{v}_{1}, \zeta_{1}\right) \\
& \quad+j\left(\boldsymbol{g}_{2}, \boldsymbol{w}_{2}, \boldsymbol{v}_{1}, \zeta_{2}\right)-j\left(\boldsymbol{g}_{2}, \boldsymbol{w}_{2}, \boldsymbol{v}_{2}, \zeta_{2}\right) \\
& \quad+\left(\boldsymbol{f}_{1}-\boldsymbol{f}_{2}, \boldsymbol{v}_{1}-\boldsymbol{v}_{2}\right)_{V}+\left(\boldsymbol{\eta}_{2}-\boldsymbol{\eta}_{1}, \boldsymbol{\varepsilon}\left(\boldsymbol{v}_{1}-\boldsymbol{v}_{2}\right)\right)_{\mathcal{H}} .
\end{aligned}
$$


Then by using (3.23), (3.12), the conditions (3.13), (3.14) and (3.15) we obtain

$$
\begin{aligned}
& m_{\mathcal{A}}\left|\boldsymbol{v}_{1}-\boldsymbol{v}_{2}\right|_{V} \leq L_{\nu}\left(1+\mu^{*}\right) c_{0}^{2}\left|\boldsymbol{g}_{1}-\boldsymbol{g}_{2}\right|_{V}+c_{0}^{2} p_{\nu}^{*} L_{\mu}\left|\boldsymbol{w}_{1}-\boldsymbol{w}_{2}\right|_{V} \\
& +\left(L_{\nu}+L_{\mu} p_{\nu}^{*}+L_{\nu} \mu^{*}\right) c_{0}\left|\zeta_{1}-\zeta_{2}\right|_{L^{2}\left(\Gamma_{3}\right)}+\left|\boldsymbol{\eta}_{1}-\boldsymbol{\eta}_{2}\right|_{\mathcal{H}}+\left|\boldsymbol{f}_{1}-\boldsymbol{f}_{2}\right|_{V} .
\end{aligned}
$$

This inequality and the regularity of the functions $\boldsymbol{f}, \boldsymbol{g}, \boldsymbol{w}, \zeta$ and $\boldsymbol{\eta}$ show that $\boldsymbol{v}_{\zeta \eta g w} \in C(0, T ; V)$. From assumption (3.13), the relation (4.5) we have

$$
\left|\boldsymbol{\sigma}_{1}-\boldsymbol{\sigma}_{2}\right|_{\mathcal{H}} \leq c\left(\left|\boldsymbol{v}_{1}-\boldsymbol{v}_{2}\right|_{V}+\left|\boldsymbol{\eta}_{1}-\boldsymbol{\eta}_{2}\right|_{\mathcal{H}}\right) .
$$

We have

$$
\operatorname{Div}_{\zeta \eta g w}(t)+\boldsymbol{f}_{0}(t)=\mathbf{0} .
$$

The regularity of the functions $\boldsymbol{\eta}, \boldsymbol{v}, \boldsymbol{f}_{0}$ and the relations (4.10), (4.11) show that $\boldsymbol{\sigma}_{\zeta \eta g w} \in C\left(0, T ; \mathcal{H}_{1}\right)$.

Let $\zeta \in C\left(0, T ; L^{2}\left(\Gamma_{3}\right)\right), \boldsymbol{g} \in C(0, T ; V)$ and $\boldsymbol{\eta} \in C(0, T ; \mathcal{H})$ be given. We consider the operator $\Lambda_{\zeta \eta g}: C(0, T ; V) \rightarrow C(0, T ; V)$ defined by

$$
\Lambda_{\zeta \eta g} \boldsymbol{w}=\boldsymbol{v}_{\zeta \eta g w} .
$$

We have the following result.

Lemma 2. The operator $\Lambda_{\zeta \eta g}$ has a unique fixed point $\boldsymbol{w}_{\zeta \eta g} \in C(0, T ; V)$.

Proof. $\quad$ Let $\boldsymbol{w}_{1}, \boldsymbol{w}_{2} \in C(0, T ; V)$. We use the notation $\boldsymbol{v}_{i}=\boldsymbol{v}_{\zeta \eta g w_{i}}$ for $i=1,2$. From the definition (4.12) we have

$$
\left|\Lambda_{\zeta \eta g} \boldsymbol{w}_{1}(t)-\Lambda_{\zeta \eta g} \boldsymbol{w}_{2}(t)\right|_{V}=\left|\boldsymbol{v}_{1}(t)-\boldsymbol{v}_{2}(t)\right|_{V} \forall t \in[0, T]
$$

Using similar arguments as those used in the proof of (4.9) we find

$$
m_{\mathcal{A}}\left|\boldsymbol{v}_{1}(t)-\boldsymbol{v}_{2}(t)\right|_{V} \leq c_{0}^{2} p_{\nu}^{*} L_{\mu}\left|\boldsymbol{w}_{1}(t)-\boldsymbol{w}_{2}(t)\right|_{V} \forall t \in[0, T]
$$

Keeping in mind that $c_{0}^{2} p_{\nu}^{*} L_{\mu}<m_{\mathcal{A}}$, the two inequalities shows that the operator $\Lambda_{\zeta \eta g}$ is a contraction in the Banach space $C(0, T ; V)$, which concludes the proof.

In what follows we denote by $\boldsymbol{w}_{\zeta \eta g}$ the fixed point given in Lemma 2 and let $\boldsymbol{v}_{\zeta \eta g} \in C(0, T ; V)$ be the function defined by $\boldsymbol{v}_{\zeta \eta g}=\boldsymbol{v}_{\zeta \eta g w_{\zeta \eta g}}$. We have $\Lambda_{\zeta \eta g} \boldsymbol{w}_{\zeta \eta g}=\boldsymbol{w}_{\zeta \eta g}$ and $\Lambda_{\zeta \eta g} \boldsymbol{w}_{\zeta \eta g}=\boldsymbol{v}_{\zeta \eta g w_{\zeta \eta g}}$ it follows that $\boldsymbol{w}_{\zeta \eta g}=\boldsymbol{v}_{\zeta \eta g}$. Therefore, choosing $\boldsymbol{w}=\boldsymbol{w}_{\zeta \eta g}$ in (4.8) and for all $\boldsymbol{v} \in V, t \in[0, T]$, we see that $\boldsymbol{v}_{\zeta \eta g}$ satisfies

$$
\begin{aligned}
& \left(A \boldsymbol{v}_{\zeta \eta g}(t), \boldsymbol{v}-\boldsymbol{v}_{\zeta \eta g}(t)\right)_{V}+j\left(\boldsymbol{g}(t), \boldsymbol{v}_{\zeta \eta g}(t), \boldsymbol{v}, \zeta(t)\right) \\
& \quad-j\left(\boldsymbol{g}(t), \boldsymbol{v}_{\zeta \eta g}(t), \boldsymbol{v}_{\zeta \eta g}(t), \zeta(t)\right) \geqslant\left(\boldsymbol{F}(t), \boldsymbol{v}-\boldsymbol{v}_{\zeta \eta g}(t)\right)_{V} .
\end{aligned}
$$

We denote by $\boldsymbol{u}_{\zeta \eta g} \in C^{1}(0, T ; V)$ the function

$$
\boldsymbol{u}_{\zeta \eta g}(t)=\boldsymbol{u}_{0}+\int_{0}^{t} \boldsymbol{v}_{\zeta \eta g}(s) d s \quad \forall t \in[0, T],
$$


and define the operator $\Lambda_{\zeta \eta}: C(0, T ; V) \rightarrow C(0, T ; V)$ by

$$
\Lambda_{\zeta \eta} \boldsymbol{g}=\boldsymbol{u}_{\zeta \eta g} .
$$

Lemma 3. The operator $\Lambda_{\zeta \eta}$ has a unique fixed point $\boldsymbol{g}_{\zeta \eta} \in C(0, T ; V)$.

Proof. Let $\boldsymbol{g}_{1}, \boldsymbol{g}_{2} \in C(0, T ; V)$. We use the notation $\boldsymbol{v}_{i}=\boldsymbol{v}_{\zeta \eta g_{i}}$ and $\boldsymbol{u}_{i}=\boldsymbol{u}_{\zeta \eta g_{i}}$ for $i=1,2$. Using (4.14) and the estimates in the proof of Lemma 1 yield for all $s \in[0, T],\left(m_{\mathcal{A}}-p_{\nu}^{*} L_{\mu} c_{0}^{2}\right)\left|\boldsymbol{v}_{1}(s)-\boldsymbol{v}_{2}(s)\right|_{V} \leq c_{0}^{2} L_{\nu}\left(1+\mu^{*}\right)\left|\boldsymbol{g}_{1}(s)-\boldsymbol{g}_{2}(s)\right|_{V}$. Using now (4.14)-(4.15) we obtain for all $t \in[0, T]$,

$$
\left|\Lambda_{\zeta \eta} \boldsymbol{g}_{1}(t)-\Lambda_{\zeta \eta} \boldsymbol{g}_{2}(t)\right|_{V} \leq \frac{c_{0}^{2} L_{\nu}\left(1+\mu^{*}\right)}{\left(m_{\mathcal{A}}-p_{\nu}^{*} L_{\mu} c_{0}^{2}\right)} \int_{0}^{t}\left|\boldsymbol{g}_{1}(s)-\boldsymbol{g}_{2}(s)\right|_{V} d s .
$$

By reiterating this inequality $m$ times, we obtain that a power of $\Lambda_{\zeta \eta}$ is a contraction mapping on $C(0, T ; V)$, which concludes the proof.

Problem $\mathbf{P} \mathbf{V}_{\zeta \eta}$. Find a displacement field $\boldsymbol{u}_{\zeta \eta}:[0, T] \rightarrow V$ such that for all $t \in[0, T]$,

$$
\begin{aligned}
& \left(\mathcal{A} \boldsymbol{\varepsilon}\left(\dot{\boldsymbol{u}}_{\zeta \eta}(t)\right), \boldsymbol{\varepsilon}\left(\boldsymbol{v}-\dot{\boldsymbol{u}}_{\zeta \eta}(t)\right)_{\mathcal{H}}+\left(\boldsymbol{\eta}(t), \boldsymbol{\varepsilon}\left(\boldsymbol{v}-\dot{\boldsymbol{u}}_{\zeta \eta}(t)\right)_{\mathcal{H}}\right.\right. \\
& +j\left(\boldsymbol{u}_{\zeta \eta}(t), \dot{\boldsymbol{u}}_{\zeta \eta}(t), \boldsymbol{v}, \zeta(t)\right)-j\left(\boldsymbol{u}_{\zeta \eta}(t), \dot{\boldsymbol{u}}_{\zeta \eta}(t), \dot{\boldsymbol{u}}_{\zeta \eta}(t), \zeta(t)\right) \\
& \geq\left(\boldsymbol{f}(t), \boldsymbol{v}-\dot{\boldsymbol{u}}_{\zeta \eta}(t)\right)_{V} \forall \boldsymbol{v} \in V \\
& \boldsymbol{u}_{\zeta \eta}(0)=\boldsymbol{u}_{0}
\end{aligned}
$$

In the study of the problem $\mathrm{PV}_{\zeta \eta}$ we have the following result.

Lemma 4. $P V_{\zeta \eta}$ has a unique solution satisfying the regularity (4.1).

Proof. For each $\zeta \in C\left(0, T ; L^{2}\left(\Gamma_{3}\right)\right)$ and $\boldsymbol{\eta} \in C(0, T ; \mathcal{H})$, we denote by $\boldsymbol{g}_{\zeta \eta} \in$ $C(0, T ; V)$ be the fixed point guaranted by Lemma 3 and let $\boldsymbol{u}_{\zeta \eta}$ be the function defined by (4.14), for $\boldsymbol{g}=\boldsymbol{g}_{\zeta \eta}$. We have $\Lambda_{\zeta \eta} \boldsymbol{g}_{\zeta \eta}=\boldsymbol{g}_{\zeta \eta}$. From (4.14) and (4.15) it follows that $\boldsymbol{u}_{\zeta \eta}=\boldsymbol{g}_{\zeta \eta}$. Therefore, taking $\boldsymbol{g}=\boldsymbol{g}_{\zeta \eta}$ in (4.13) and using (4.7) and (4.14) we see that $\boldsymbol{u}_{\zeta \eta}$ is the unique solution to problem $\mathrm{PV}_{\zeta \eta}$ satisfying the regularity expressed in (4.1).

In the second step, let $\zeta \in C\left(0, T ; L^{2}\left(\Gamma_{3}\right)\right)$ and $\boldsymbol{\eta} \in C(0, T ; \mathcal{H})$, we use the displacement field $\boldsymbol{u}_{\zeta \eta}$ defined in (4.14) and consider the following variational problem.

Problem $\mathbf{Q V}_{\zeta \eta}$. Find the electric potential field $\varphi_{\zeta \eta}:[0, T] \rightarrow W$ such that $\forall t \in[0, T]$

$$
\begin{aligned}
& \left(\boldsymbol{B} \nabla \varphi_{\zeta \eta}(t), \nabla \phi\right)_{H}+\left(\gamma\left(\boldsymbol{u}_{\zeta \eta}(t), \varphi_{\zeta \eta}(t), \zeta(t)\right), \phi\right)_{W} \\
& -\left(\mathcal{E} \boldsymbol{\varepsilon}\left(\boldsymbol{u}_{\zeta \eta}(t)\right), \nabla \phi\right)_{H}=(q(t), \phi)_{W} \quad \forall \phi \in W,
\end{aligned}
$$

we have the following result. 
Lemma 5. $Q V_{\zeta \eta}$ has a unique solution $\varphi_{\zeta \eta}$ which satisfies the regularity expressed in (4.2). Moreover, if $\varphi_{\zeta \eta_{1}}$ and $\varphi_{\zeta \eta_{2}}$ are the solutions of (4.17) corresponding to $\boldsymbol{\eta}_{1}, \boldsymbol{\eta}_{2} \in C(0, T ; \mathcal{H})$ then, there exists $c>0$, such that

$$
\left|\varphi_{\zeta \eta_{1}}(t)-\varphi_{\zeta \eta_{2}}(t)\right|_{W} \leq c\left|\boldsymbol{u}_{\zeta \eta_{1}}(t)-\boldsymbol{u}_{\zeta \eta_{2}}(t)\right|_{V} \forall t \in[0, T]
$$

Proof. Let $t \in[0, T]$. We use Riesz representation theorem to define the operator $A_{\zeta \eta}(t): W \rightarrow W$ by

$$
\begin{aligned}
& \left(A_{\zeta \eta}(t) \varphi, \phi\right)_{W}=(\boldsymbol{B} \nabla \varphi, \nabla \phi)_{H}-\left(\mathcal{E} \varepsilon\left(\boldsymbol{u}_{\zeta \eta}(t)\right), \nabla \phi\right)_{H} \\
& +\left(\gamma\left(\boldsymbol{u}_{\zeta \eta}(t), \varphi, \zeta(t)\right), \phi\right)_{W} \forall \varphi, \phi \in W .
\end{aligned}
$$

Let $\varphi_{1}, \varphi_{2} \in W$. Assumption (3.16)-(3.18) and the definition (3.24) imply $A_{\zeta \eta}(t)$ is a strongly monotone and Lipschitz continuous operator on $W$. We deduce that there exists a unique element $\varphi_{\zeta \eta}(t) \in W$ such that $A_{\zeta \eta}(t) \varphi_{\zeta \eta}(t)=$ $q(t)$. We conclude that $\varphi_{\zeta \eta}$ is the unique solution of $\mathrm{QV}_{\zeta \eta}$.

We show next that $\varphi_{\zeta \eta} \in C(0, T ; W)$. Let $t_{1}, t_{2} \in[0, T]$ and denote $\varphi_{\zeta \eta}\left(t_{i}\right)=$ $\varphi_{i}, u_{\zeta \eta \nu}\left(t_{i}\right)=u_{i \nu}, \boldsymbol{u}_{\zeta \eta}\left(t_{i}\right)=\boldsymbol{u}_{i}, q\left(t_{i}\right)=q_{i}, \zeta\left(t_{i}\right)=\zeta_{i}$ for $i=1,2$. Using (4.17), (3.16), (3.17) and the definition (3.24) we find

$$
\begin{aligned}
& m_{B}\left|\varphi_{1}-\varphi_{2}\right|_{W} \leq\left(c_{\mathcal{E}}+L_{\psi} L a_{0} c_{0}\right)\left|\boldsymbol{u}_{1}-\boldsymbol{u}_{2}\right|_{V}+\left|q_{1}-q_{2}\right|_{W} \\
& +L_{\psi} L a_{0}\left|\zeta_{1}-\zeta_{2}\right|_{L^{2}\left(\Gamma_{3}\right)}+N_{\psi} a_{0}^{2}\left|\varphi_{1}-\varphi_{2}\right|_{W}
\end{aligned}
$$

where $c_{\mathcal{E}}$ is a positive constant which depends on the piezoelectric tensor $\mathcal{E}$. It follows from inequality (4.19) and the fact that $N_{\psi}<\frac{m_{B}}{a_{0}^{2}}$ that

$$
\left|\varphi_{1}-\varphi_{2}\right|_{W} \leq c\left(\left|\boldsymbol{u}_{1}-\boldsymbol{u}_{2}\right|_{V}+\left|q_{1}-q_{2}\right|_{W}+\left|\zeta_{1}-\zeta_{2}\right|_{L^{2}\left(\Gamma_{3}\right)}\right) \text {. }
$$

Since $\boldsymbol{u} \in C^{1}(0, T ; V), q \in C(0, T ; W), \zeta \in C\left(0, T ; L^{2}\left(\Gamma_{3}\right)\right)$ the inequality (4.20) implies that $\varphi_{\zeta \eta} \in C(0, T ; W)$.

Let $\boldsymbol{\eta}_{1}, \boldsymbol{\eta}_{2} \in C(0, T ; \mathcal{H})$ and denote $\varphi_{\zeta \eta_{i}}=\varphi_{i}, \boldsymbol{u}_{\zeta \eta_{i}}=\boldsymbol{u}_{i}$ for $i=1,2$. We use (4.17) and arguments similar to those used in the proof of (4.19) to obtain

$$
\begin{aligned}
& m_{B}\left|\varphi_{1}(t)-\varphi_{2}(t)\right|_{W} \leq N_{\psi} a_{0}^{2}\left|\varphi_{1}(t)-\varphi_{2}(t)\right|_{W} \\
& +\left(c_{\mathcal{E}}+L_{\psi} L a_{0} c_{0}\right)\left|\boldsymbol{u}_{1}(t)-\boldsymbol{u}_{2}(t)\right|_{V},
\end{aligned}
$$

for all $t \in[0, T]$. This inequality, combined with the fact that $N_{\psi}<\frac{m_{B}}{a_{0}^{2}}$ leads to (4.18) which concludes the proof.

In the third step, we let $\theta \in C\left(0, T ; L^{2}(\Omega)\right)$ be given and consider the following variational problem for the damage field.

Problem $\mathbf{P} V_{\theta}$. Find a damage field $\beta_{\theta}:[0, T] \rightarrow H^{1}(\Omega)$ such that

$$
\begin{aligned}
& \beta_{\theta}(t) \in K,\left(\dot{\beta}_{\theta}(t), \xi-\beta_{\theta}(t)\right)_{L^{2}(\Omega)}+a\left(\beta_{\theta}(t), \xi-\beta_{\theta}(t)\right) \\
& \geq\left(\theta(t), \xi-\beta_{\theta}(t)\right)_{L^{2}(\Omega)} \quad \forall \xi \in K, \text { a.e. } t \in(0, T), \quad \beta_{\theta}(0)=\beta_{0} .
\end{aligned}
$$

Lemma 6. Problem $P V_{\theta}$ has a unique solution $\beta_{\theta}$ which satisfies $\beta_{\theta} \in H^{1}\left(0, T ; L^{2}(\Omega)\right) \cap L^{2}\left(0, T ; H^{1}(\Omega)\right)$. 
Proof. To solve $P V_{\theta}$, we use the standard result for parabolic variational inequalities ( see for example the reference [23, p.47]).

Finally, we consider the operator $\Lambda_{\zeta}: C\left(0, T ; \mathcal{H} \times L^{2}(\Omega)\right) \rightarrow C(0, T ; \mathcal{H} \times$ $\left.L^{2}(\Omega)\right)$ defined by

$$
\begin{aligned}
& \Lambda_{\zeta}(\boldsymbol{\eta}, \theta)(t)=\left(\Lambda_{\zeta}^{(1)}(\boldsymbol{\eta}, \theta)(t), \Lambda_{\zeta}^{(2)}(\boldsymbol{\eta}, \theta)(t)\right) \in \mathcal{H} \times L^{2}(\Omega) \\
& \Lambda_{\zeta}^{(1)}(\boldsymbol{\eta}, \theta)(t)=\mathcal{F}\left(\varepsilon\left(\boldsymbol{u}_{\zeta \eta}\right), \beta_{\theta}\right)+\mathcal{E}^{*} \nabla \varphi_{\zeta \eta}(t)+\int_{0}^{t} M(t-s) \varepsilon\left(\boldsymbol{u}_{\zeta \eta}(s)\right) d s, \\
& \Lambda_{\zeta}^{(2)}(\boldsymbol{\eta}, \theta)(t)=S\left(\varepsilon\left(\boldsymbol{u}_{\zeta \eta}(t)\right), \beta_{\theta}(t)\right)
\end{aligned}
$$

Here, for every $(\boldsymbol{\eta}, \theta) \in C\left(0, T ; \mathcal{H} \times L^{2}(\Omega)\right), \boldsymbol{u}_{\zeta \eta}, \varphi_{\zeta \eta}$ and $\beta_{\theta}$ represent the displacement field, the electric potential field and the damage field obtained in Lemmas 4, 5 and 6 respectively.

By using similar arguments to those used in the Lemma 4.8 in [14], we have the following result.

Lemma 7. The operator $\Lambda_{\zeta}$ has a unique fixed point $\left(\boldsymbol{\eta}_{\zeta}, \theta_{\zeta}\right) \in C(0, T ; \mathcal{H} \times$ $\left.L^{2}(\Omega)\right)$.

Let $\zeta \in C\left(0, T ; L^{2}\left(\Gamma_{3}\right)\right)$. In the fourth step we consider the following variational problem.

Problem $\mathbf{P} \mathbf{V}_{\zeta}$. Find a displacement field $\boldsymbol{u}_{\zeta}:[0, T] \rightarrow V$, a stress field $\boldsymbol{\sigma}_{\zeta}:[0, T] \rightarrow \mathcal{H}_{1}$, an electric potential field $\varphi_{\zeta}:[0, T] \rightarrow W$, an electric displacement field $\boldsymbol{D}_{\zeta}:[0, T] \rightarrow \mathcal{W}$ and a damage field $\beta_{\zeta}:[0, T] \rightarrow H^{1}(\Omega)$ such that

$$
\begin{aligned}
& \boldsymbol{\sigma}_{\zeta}(t)=\mathcal{A} \boldsymbol{\varepsilon}\left(\dot{\boldsymbol{u}}_{\zeta}(t)\right)+\mathcal{F}\left(\varepsilon\left(\boldsymbol{u}_{\zeta}(t)\right), \beta_{\zeta}(t)\right) \\
& +\mathcal{E}^{*} \nabla \varphi_{\zeta}(t)+\int_{0}^{t} M(t-s) \varepsilon\left(\boldsymbol{u}_{\zeta}(s)\right) d s, t \in[0, T] \\
& \left(\boldsymbol{\sigma}_{\zeta}(t), \boldsymbol{\varepsilon}\left(\boldsymbol{v}-\dot{\boldsymbol{u}}_{\zeta}(t)\right)\right)_{\mathcal{H}}+j\left(\boldsymbol{u}_{\zeta}(t), \dot{\boldsymbol{u}}_{\zeta}(t), \boldsymbol{v}, \zeta(t)\right) \\
& \quad-j\left(\boldsymbol{u}_{\zeta}(t), \dot{\boldsymbol{u}}_{\zeta}(t), \dot{\boldsymbol{u}}_{\zeta}(t), \zeta(t)\right) \geq\left(\boldsymbol{f}(t), \boldsymbol{v}-\dot{\boldsymbol{u}}_{\zeta}(t)\right)_{V} \quad \forall \boldsymbol{v} \in V, t \in[0, T], \\
& \boldsymbol{D}_{\zeta}(t)=\mathcal{E} \boldsymbol{\varepsilon}\left(\boldsymbol{u}_{\zeta}(t)\right)-\boldsymbol{B} \nabla \varphi_{\zeta}(t), t \in[0, T] \\
& \left(\boldsymbol{D}_{\zeta}(t), \nabla \phi\right)_{H}=\left(\gamma\left(\boldsymbol{u}_{\zeta}(t), \varphi_{\zeta}(t), \zeta(t)\right), \phi\right)_{W} \\
& \quad-(q(t), \phi)_{W} \forall \phi \in W, t \in[0, T] \\
& \beta_{\zeta}(t) \in K, \quad\left(\dot{\beta}_{\zeta}(t), \xi-\beta_{\zeta}(t)\right)_{L^{2}(\Omega)}+a\left(\beta_{\zeta}(t), \xi-\beta_{\zeta}(t)\right) \\
& \geq\left(S\left(\varepsilon\left(\boldsymbol{u}_{\zeta}(t)\right), \beta_{\zeta}(t)\right), \xi-\beta_{\zeta}(t)\right)_{L^{2}(\Omega)} \forall \xi \in K, \text { a.e. } t \in(0, T), \\
& \boldsymbol{u}_{\zeta}(0)=\boldsymbol{u}_{0}, \beta_{\zeta}(0)=\beta_{0} .
\end{aligned}
$$

Lemma 8. Problem $P V_{\zeta}$ has a unique solution $\left(\boldsymbol{u}_{\zeta}, \boldsymbol{\sigma}_{\zeta}, \varphi_{\zeta}, \boldsymbol{D}_{\zeta}, \beta_{\zeta}\right)$ satisfying $(4.1)-(4.3)$.

Proof. Let $\left(\boldsymbol{\eta}_{\zeta}, \theta_{\zeta}\right) \in C\left(0, T ; \mathcal{H} \times L^{2}(\Omega)\right)$ be the fixed point of $\Lambda_{\zeta}$ defined by (4.21)-(4.23) and denote $\boldsymbol{u}_{\zeta}=\boldsymbol{u}_{\zeta \eta_{\zeta}}, \varphi_{\zeta}=\varphi_{\zeta \eta_{\zeta}}, \beta_{\zeta}=\beta_{\theta_{\zeta}}$, be the solutions to 
problems $P V_{\zeta \eta}, Q V_{\zeta \eta}$ and $P V_{\theta}$ obtained in Lemmas 4, 5 and 6 respectively for $(\boldsymbol{\eta}, \theta)=\left(\boldsymbol{\eta}_{\zeta}, \theta_{\zeta}\right)$. Let

$$
\begin{aligned}
& \boldsymbol{\sigma}_{\zeta}(t)=\mathcal{A} \boldsymbol{\varepsilon}\left(\dot{\boldsymbol{u}}_{\zeta}(t)\right)+\mathcal{F} \boldsymbol{\varepsilon}\left(\boldsymbol{u}_{\zeta}(t), \beta_{\zeta}(t)\right)+\mathcal{E}^{*} \nabla \varphi_{\zeta}(t) \\
& +\int_{0}^{t} M(t-s) \boldsymbol{\varepsilon}\left(\boldsymbol{u}_{\zeta}(s)\right) d s, \quad \forall t \in[0, T] \\
& \boldsymbol{D}_{\zeta}(t)=\mathcal{E} \boldsymbol{\varepsilon}\left(\boldsymbol{u}_{\zeta}(t)\right)-\boldsymbol{B} \nabla \varphi_{\zeta}(t), \forall t \in[0, T] .
\end{aligned}
$$

Equations $\Lambda_{\zeta}^{(1)}\left(\boldsymbol{\eta}_{\zeta}, \theta_{\zeta}\right)=\boldsymbol{\eta}_{\zeta}$ and $\Lambda_{\zeta}^{(2)}\left(\boldsymbol{\eta}_{\zeta}, \theta_{\zeta}\right)=\theta_{\zeta}$, combined with (4.22) and (4.23) shows that $\left(\boldsymbol{u}_{\zeta}, \boldsymbol{\sigma}_{\zeta}, \varphi_{\zeta}, \boldsymbol{D}_{\zeta}, \beta_{\zeta}\right)$ satisfies (4.24)-(4.25). Next, (4.26) and the regularities (4.1)-(4.3) follow from Lemmas 4, 5 and 6 and assumptions on $\mathcal{A}, \mathcal{F}, M$ and $\mathcal{E}$ which concludes the existence part of the Lemma 8.

The uniqueness part of Lemma 8 is a consequence of the uniqueness of the fixed point of the operator $\Lambda_{\zeta}$ defined by $(4.21)-(4.23)$ and the unique solvability of problems $P V_{\zeta \eta_{\zeta}}, Q V_{\zeta \eta_{\zeta}}$ and $P V_{\theta_{\zeta}}$.

Let us now consider the operator $\mathcal{T}: C\left(0, T ; L^{2}\left(\Gamma_{3}\right)\right) \rightarrow C\left(0, T ; L^{2}\left(\Gamma_{3}\right)\right)$ defined for all $t \in[0, T]$ by

$$
\mathcal{T} \zeta(t)=k_{1} \int_{0}^{t} \mu\left(\zeta,\left|\dot{\boldsymbol{u}}_{\tau}-\boldsymbol{v}^{*}\right|\right) p_{\nu}\left(u_{\nu}-h-\zeta\right) R^{*}\left(\left|\dot{\boldsymbol{u}}_{\tau}-\boldsymbol{v}^{*}\right|\right) d s .
$$

The last step in the proof of Theorem 1 is the next result.

Lemma 9. The operator $\mathcal{T}$ has a unique fixed point $\zeta^{*} \in C\left(0, T ; L^{2}\left(\Gamma_{3}\right)\right)$.

Proof. Let $\zeta_{1}, \zeta_{2} \in C\left(0, T ; L^{2}\left(\Gamma_{3}\right)\right)$ and denote by $\left(\boldsymbol{u}_{i}, \boldsymbol{\sigma}_{i}, \varphi_{i}, \boldsymbol{D}_{i}\right), i=1,2$, the solutions to problem $P V_{\zeta}$ for $\zeta=\zeta_{i}$, i.e. $\boldsymbol{u}_{i}=\boldsymbol{u}_{\zeta_{i}}, \boldsymbol{v}_{i}=\dot{\boldsymbol{u}}_{\zeta_{i}}, \boldsymbol{\sigma}_{i}=\boldsymbol{\sigma}_{\zeta_{i}}$, $\varphi_{i}=\varphi_{\zeta_{i}}$ and $\boldsymbol{D}_{i}=\boldsymbol{D}_{\zeta_{i}}$. Moreover, we denote in the sequel by $c$ various positive constants which may depend on $k_{1}$ and $\boldsymbol{v}^{*}$. We use similar arguments that those used in the proof of the relation (4.9) to find that for all $s \in[0, T]$

$$
\left|\boldsymbol{v}_{1}(s)-\boldsymbol{v}_{2}(s)\right|_{V} \leq c\left(\left|\boldsymbol{u}_{1}(s)-\boldsymbol{u}_{2}(s)\right|_{V}+\left|\zeta_{1}(s)-\zeta_{2}(s)\right|_{L^{2}\left(\Gamma_{3}\right)}\right) \text {. }
$$

Using the inequality $2 a b \leq a^{2}+b^{2}$ and integrating this inequality with respect to time, we obtain

$$
\int_{0}^{t}\left|\boldsymbol{v}_{1}(s)-\boldsymbol{v}_{2}(s)\right|_{V}^{2} d s \leq c\left(\int_{0}^{t}\left|\boldsymbol{u}_{1}(s)-\boldsymbol{u}_{2}(s)\right|_{V}^{2} d s+\int_{0}^{t}\left|\zeta_{1}(s)-\zeta_{2}(s)\right|_{L^{2}\left(\Gamma_{3}\right)}^{2} d s\right) .
$$

Since $\boldsymbol{u}_{1}(0)=\boldsymbol{u}_{2}(0)=\boldsymbol{u}_{0}$ and using the previous inequality we obtain

$$
\begin{aligned}
& \left|\boldsymbol{u}_{1}(t)-\boldsymbol{u}_{2}(t)\right|_{V}^{2} \leq c \int_{0}^{t}\left|\boldsymbol{v}_{1}(s)-\boldsymbol{v}_{2}(s)\right|_{V}^{2} d s \\
& \leq c \int_{0}^{t}\left|\boldsymbol{u}_{1}(s)-\boldsymbol{u}_{2}(s)\right|_{V}^{2} d s+c \int_{0}^{t}\left|\zeta_{1}(s)-\zeta_{2}(s)\right|_{L^{2}\left(\Gamma_{3}\right)}^{2} d s .
\end{aligned}
$$

Applying Gronwall inequality, we deduce that

$$
\left|\boldsymbol{u}_{1}(t)-\boldsymbol{u}_{2}(t)\right|_{V}^{2} \leq c \int_{0}^{t}\left|\zeta_{1}(s)-\zeta_{2}(s)\right|_{L^{2}\left(\Gamma_{3}\right)}^{2} d s
$$


It follows from (4.29) and the previous inequality that

$$
\begin{aligned}
\int_{0}^{t}\left|\boldsymbol{v}_{1}(s)-\boldsymbol{v}_{2}(s)\right|_{V}^{2} d s \leq & c\left(\int_{0}^{t} \int_{0}^{s}\left|\zeta_{1}(r)-\zeta_{2}(r)\right|_{L^{2}\left(\Gamma_{3}\right)}^{2} d r d s\right. \\
& \left.+\int_{0}^{t}\left|\zeta_{1}(s)-\zeta_{2}(s)\right|_{L^{2}\left(\Gamma_{3}\right)}^{2} d s\right) .
\end{aligned}
$$

Since $s \leq t$

$$
\begin{aligned}
\int_{0}^{t} \int_{0}^{s}\left|\zeta_{1}(r)-\zeta_{2}(r)\right|_{L^{2}\left(\Gamma_{3}\right)}^{2} d r d s & \leq c \int_{0}^{t} \int_{0}^{t}\left|\zeta_{1}(r)-\zeta_{2}(r)\right|_{L^{2}\left(\Gamma_{3}\right)}^{2} d r d s \\
& =c \int_{0}^{t}\left|\zeta_{1}(r)-\zeta_{2}(r)\right|_{L^{2}\left(\Gamma_{3}\right)}^{2} d r \int_{0}^{t} d s
\end{aligned}
$$

Then

$$
\int_{0}^{t}\left|\boldsymbol{v}_{1}(s)-\boldsymbol{v}_{2}(s)\right|_{V}^{2} d s \leq c \int_{0}^{t}\left|\zeta_{1}(s)-\zeta_{2}(s)\right|_{L^{2}\left(\Gamma_{3}\right)}^{2} d s .
$$

On the other hand, since

$$
\mathcal{T} \zeta_{i}(t)=k_{1} \int_{0}^{t} \mu\left(\zeta_{i}(s),\left|\boldsymbol{v}_{i \tau}(s)-\boldsymbol{v}^{*}\right|\right) p_{\nu}\left(u_{i \nu}(s)-h-\zeta_{i}(s)\right) R^{*}\left(\left|\boldsymbol{v}_{i \tau}(s)-\boldsymbol{v}^{*}\right|\right) d s
$$

for $i=1,2$, we use similar arguments that those used in the proof of the relation (4.9) to obtain that for $t \in[0, T]$

$$
\left|\mathcal{T} \zeta_{1}(t)-\mathcal{T} \zeta_{2}(t)\right|_{L^{2}\left(\Gamma_{3}\right)}^{2} \leq c \int_{0}^{t}\left(\left|\boldsymbol{v}_{1}(s)-\boldsymbol{v}_{2}(s)\right|_{V}^{2}+\left|\zeta_{1}(s)-\zeta_{2}(s)\right|_{L^{2}\left(\Gamma_{3}\right)}^{2}\right) d s
$$

We substitute (4.30) in the previous inequality to find

$$
\left|\mathcal{T} \zeta_{1}(t)-\mathcal{T} \zeta_{2}(t)\right|_{L^{2}\left(\Gamma_{3}\right)}^{2} \leq c \int_{0}^{t}\left|\zeta_{1}(s)-\zeta_{2}(s)\right|_{L^{2}\left(\Gamma_{3}\right)}^{2} d s
$$

Reiterating this inequality $m$ times leads to

$$
\left|\mathcal{T}^{m} \zeta_{1}-\mathcal{T}^{m} \zeta_{2}\right|_{C\left(0, T ; L^{2}\left(\Gamma_{3}\right)\right)}^{2} \leq \frac{(c T)^{m}}{m !}\left|\zeta_{1}-\zeta_{2}\right|_{C\left(0, T ; L^{2}\left(\Gamma_{3}\right)\right)}^{2}
$$

Therefore, for $m$ large enough, $\mathcal{T}^{m}$ is a contractive operator on the Banach space $C\left(0, T ; L^{2}\left(\Gamma_{3}\right)\right)$. The operator $\mathcal{T}$ has a unique fixed point $\zeta^{*} \in C\left(0, T ; L^{2}\left(\Gamma_{3}\right)\right)$.

Now we have all ingredients to prove Theorem 1.

Proof. Let $\zeta^{*}$ be the fixed point of the operator $\mathcal{T}$ given by (4.28). With $(4.24)-(4.27)$ and $(4.28)$ it easy to verify that $\left(\boldsymbol{u}_{\zeta^{*}}, \boldsymbol{\sigma}_{\zeta^{*}}, \varphi_{\zeta^{*}}, \boldsymbol{D}_{\zeta^{*}}, \beta_{\zeta^{*}}, \zeta^{*}\right)$ is the unique solution to the problem $P V$ satisfying the regularities (4.1)-(4.4). 


\section{Acknowledgements}

This work has been realized thanks to the: Direction Générale de la Recherche Scientifique et du Développement Technologique "DGRSDT". MESRS Algeria.

\section{References}

[1] K.T. Andrews, M. Shillor, S. Wright and A. Klarbring. A dynamic thermoviscoelastic contact problem with friction and wear. International journal of engineering science, 35(14):1291-1309, 1997. https://doi.org/10.1016/S00207225(97)87426-5.

[2] R.C. Batra and J.S. Yang. Saint-Venant's principle in linear piezoelectricity. Journal of Elasticity, 38(2):209-218, 1995. https://doi.org/10.1007/BF00042498.

[3] P. Bisegna, F. Maceri and F. Lebon. The unilateral frictional contact of a piezoelectric body with a rigid support. In Contact mechanics, pp. 347-354. Springer Publishing, 2002. https://doi.org/10.1007/978-94-017-1154-8_37.

[4] M. Dalah and M. Sofonea. Antiplane frictional contact of electro-viscoelastic cylinders. Electronic Journal of Differential Equations (EJDE)[electronic only], 2007(161):1-14, 2007.

[5] G. Duvant and J.L. Lions. Inequalities in mechanics and physics, volume 219. Springer Science \& Business Media, 2012.

[6] W. Han and M. Sofonea. Evolutionary variational inequalities arising in viscoelastic contact problems. SIAM Journal on Numerical Analysis, 38(2):556579, 2000. https://doi.org/10.1137/S0036142998347309.

[7] T. Ikeda. Fundamentals of piezoelectricity. Oxford University Press, Oxford, 1990.

[8] L. Kaki and M. Denche. Variational analysis for some frictional contact problems. Boletim da Sociedade Paranaense de Matemática, 38(7):21-36, 2020. https://doi.org/10.5269/bspm.v38i7.44258.

[9] Z. Lerguet, M. Shillor and M. Sofonea. A frictional contact problem for an electro-viscoelastic body. Electronic Journal of Differential Equations (EJDE) [electronic only], 2007(170):1-16, 2007.

[10] F. Maceri and P. Bisegna. The unilateral frictionless contact of a piezoelectric body with a rigid support. Mathematical and Computer Modelling, 28(4-8):1928, 1998. https://doi.org/10.1016/S0895-7177(98)00105-8.

[11] J.A. C Martins and J.T. Oden. Existence and uniqueness results for dynamic contact problems with nonlinear normal and friction interface laws. Nonlinear Analysis TMA, 11(3):407-428, 1987. https://doi.org/10.1016/0362-546X(87)90055-1.

[12] S. Migórski. Hemivariational inequality for a frictional contact problem in elastopiezoelectricity. Discrete ES Continuous Dynamical Systems-B, 6(6):1339-13356, 2006. https://doi.org/10.3934/dcdsb.2006.6.1339.

[13] M. Rochdi, M. Shillor and M. Sofonea. Quasistatic viscoelastic contact with normal compliance and friction. Journal of Elasticity, 51(2):105-126, 1998. https://doi.org/10.1023/A:1007413119583.

[14] M. Selmani. A dynamic problem with adhesion and damage in electroviscoelasticity with long-term memory. JIPAM. Journal of Inequalities in Pure E6 Applied Mathematics [electronic only], 10(1):1-19, 2009. 
[15] M. Selmani. A frictional contact problem involving piezoelectric materials with long memory. Mediterranean Journal of Mathematics, 12(3):1177-1197, 2015. https://doi.org/10.1007/s00009-014-0430-1.

[16] M. Selmani et al. Frictional contact problem with wear for electro-viscoelastic materials with long memory. Bulletin of the Belgian Mathematical Society-Simon Stevin, 20(3):461-479, 2013. https://doi.org/10.36045/bbms/1378314510.

[17] M. Selmani and L. Selmani. A dynamic frictionless contact problem with adhesion and damage. Bulletin of the Polish Academy of Sciences. Mathematics, 55(1):17-34, 2007. https://doi.org/10.4064/ba55-1-3.

[18] M. Selmani and L. Selmani. A frictional contact problem with wear and damage for electro-viscoelastic materials. Applications of Mathematics, 55(2):89-109, 2010. https://doi.org/10.1007/s10492-010-0004-x.

[19] M. Shillor, M. Sofonea and J.J. Telega. Quasistatic viscoelastic contact with friction and wear diffusion. Quart. Appl. Math, 62(2):379-399, 2004. https://doi.org/10.1090/qam/2054605.

[20] M. Sofonea and R. Arhab. An electro-viscoelastic contact problem with adesion. Dynamics of Continuous Discrete and Impulsive Systems Series A, 14(4):577, 2007. https://doi.org/10.1080/00036810701286304.

[21] M. Sofonea and E. Essoufi. A piezoelectric contact problem with slip dependent coefficient of friction. Mathematical Modelling and Analysis, 9(3):229-242, 2004. https://doi.org/10.3846/13926292.2004.9637256.

[22] M. Sofonea and E. Essoufi. Quasistatic frictional contact of viscoelastic piezoelectric body. Ad. Math. Sci. Appl, 14(3):613-631, 2004.

[23] M. Sofonea, W. Han and M. Shillor. Analysis and approximation of contact problems with adhesion or damage. CRC Press, 2006. https://doi.org/10.1201/9781420034837.

[24] M. Sofonea, F. Pătrulescu and Y. Souleiman. Analysis of a contact problem with wear and unilateral constraint. Applicable Analysis, 95(11):2590-2607, 2016. https://doi.org/10.1080/00036811.2015.1102892.

[25] M. Sofonea and Y. Souleiman. Analysis of a sliding frictional contact problem with unilateral constraint. Mathematics and Mechanics of Solids, 22(3):324-342, 2015. https://doi.org/10.1177/1081286515591304.

[26] M. Sofonea and Y. Souleiman. A viscoelastic sliding contact problem with normal compliance, unilateral constraint and memory term. Mediterranean Journal of Mathematics, 13:2863-2886, 2016. https://doi.org/10.1007/s00009-015-0661-9.

[27] B. Souraya and A.A. Abdelaziz. Analysis of a dynamic contact problem for electro-viscoelastic materials. Milan Journal of Mathematics, 86(1):105-124, 2018. https://doi.org/10.1007/s00032-018-0282-4.

[28] N. Strömberg. Continuum thermodynamics of contact, friction and wear. Ph.D. Thesis. Linkoping University, Sweeden, 1995.

[29] N. Strömberg, L. Johansson and A. Klarbring. Derivation and analysis of a generalized standard model for contact, friction and wear. International Journal of Solids and Structures, 33(13):1817-1836, 1996. https://doi.org/10.1016/00207683(95)00140-9. 